\title{
North-south asymmetry in the magnetic deflection of polar coronal hole jets
}

\author{
G. Nisticò ${ }^{1}$, G. Zimbardo ${ }^{2}$, S. Patsourakos ${ }^{3}$, V. Bothmer ${ }^{4}$, and V. M. Nakariakov ${ }^{1,5,6}$ \\ ${ }^{1}$ Centre for Fusion, Space and Astrophysics, Department of Physics, University of Warwick, Coventry CV4 7AL, UK \\ e-mail: g.nistico@warwick.ac.uk \\ 2 Dipartimento di Fisica, Università della Calabria, 87036 Arcavacata di Rende, (CS), Italy \\ 3 Department of Physics, Section Astro-Geophysics, University of Ioannina, 45110 Ioannina, Greece \\ ${ }^{4}$ Institut für Astrophysik, Georg-August University of Göttingen, 37077 Göttingen, Germany \\ 5 Central Astronomical Observatory at Pulkovo of the Russian Academy of Sciences, 196140 St. Petersburg, Russia \\ ${ }^{6}$ School of Space Research, Kyung Hee University, Yongin, 446-701 Gyeonggi, Korea
}

Received 23 January 2015 / Accepted 22 July 2015

\begin{abstract}
Context. Measurements of the sunspots area, of the magnetic field in the interplanetary medium, and of the heliospheric current sheet (HCS) position, reveal a possible north-south (N-S) asymmetry in the magnetic field of the Sun. This asymmetry could cause the bending of the HCS of the order of 5-10 deg in the southward direction, and it appears to be a recurrent characteristic of the Sun during the minima of solar activity.

Aims. We study the N-S asymmetry as inferred from measurements of the deflection of polar coronal hole jets when they propagate throughout the corona.

Methods. Since the corona is an environment where the magnetic pressure is greater than the kinetic pressure $(\beta \ll 1)$, we can assume that the magnetic field controls the dynamics of plasma. On average, jets follow magnetic field lines during their propagation, highlighting their local direction. We measured the position angles at $1 R_{\odot}$ and at $2 R_{\odot}$ of 79 jets, based on the Solar TErrestrial RElations Observatory (STEREO) ultraviolet and white-light coronagraph observations during the solar minimum period March 2007-April 2008. The average jet deflection is studied both in the plane perpendicular to the line of sight and, for a reduced number of jets, in 3D space. The observed jet deflection is studied in terms of an axisymmetric magnetic field model comprising dipole $\left(g_{1}\right)$, quadrupole $\left(g_{2}\right)$, and esapole $\left(g_{3}\right)$ moments.

Results. We found that the propagation of the jets is not radial, which is in agreement with the deflection due to magnetic field lines. Moreover, the amount of the deflection is different between jets over the north and those from the south pole. A comparison of jet deflections and field line tracing shows that a ratio $g_{2} / g_{1} \simeq-0.5$ for the quadrupole and a ratio $g_{3} / g_{1} \simeq 1.6-2.0$ for the esapole can describe the field. The presence of a non-negligible quadrupole moment confirms the N-S asymmetry of the solar magnetic field for the considered period.

Conclusions. We find that the magnetic deflection of jets is larger in the north than in the south of the order of 25-40\%, with an asymmetry that is consistent with a southward deflection of the heliospheric current sheet of the order of 10 deg, consistent with that inferred from other independent datasets and instruments.
\end{abstract}

Key words. Sun: corona - Sun: magnetic fields - methods: observational

\section{Introduction}

The solar corona is an environment highly structured by the strength and the topology of magnetic fields. Even during solar minima, the corona is far from being a quiet region but it evolves on many timescales, including the solar cycle. Observations of the corona in extreme ultraviolet (EUV) and X-ray wavelengths reveal bright (dense) regions, coinciding with the presence of active regions, and extended dark (void) areas, called coronal holes. Similarly, white-light observations obtained from coronagraphs show the presence of ray-like features at higher latitudes, and helmet streamers at middle and equatorial latitudes. In astrophysics, knowledge of the solar magnetic field comes mainly from measurements of splitting atomic lines due to the Zeeman effect. This method can be applied to the radiation coming from the photosphere, allowing us to estimate the vector magnetic field, but there are not many opportunities to obtain direct measurements of the magnetic field of the corona. Indirect estimates of the coronal magnetic field are obtained from extrapolations techniques, such as the potential field source surface (PFSS) and the non-linear force-free field (NLFFF) models (Wiegelmann \& Sakurai 2012), by radiophysical methods and coronal seismology (e.g. Nakariakov \& Ofman 2001).

There are several observations suggesting a northsouth (N-S) asymmetry of the solar magnetic field during solar minima (e.g. see the discussion in Erdős \& Balogh 2010). Early evidence came from direct measurements of the photospheric magnetic field by magnetograms, which were extrapolated to the solar wind source surface by Hoeksema (1995), who showed that the magnetic field strength in the Sun's south polar cap was $60 \%$ larger than in the north polar cap during some solar minima. Then, the Ulysses spacecraft provided new insights of the N-S asymmetry: thanks to its orbit, nearly perpendicular to the ecliptic plane, Ulysses explored high-latitude regions of the heliosphere, and during 
its passage close to the Sun, the so-called fast latitude scan at about 1.4 AU, gave us measurements of the interplanetary magnetic field (Erdős \& Balogh 1998) as well as particle data. Indeed, an indication of N-S asymmetry comes from the global distribution of the solar wind speed (Tokumaru et al. 2015) and the latitudinal gradients of energetic particle fluxes (Simpson et al. 1996; Heber et al. 1996), which show an imbalance of fluxes between north and south of $6 \%-15 \%$. Moreover, Erdôs \& Balogh (2010) studied and compared magnetic field data of Ulysses during the first latitude scan (coincident with the minimum of solar cycle 22 in 1994-1995) and the third scan (minimum of solar cycle 23 in 2007-2008). They found that the radial component of the magnetic field (normalised to $1 \mathrm{AU}$ ) of the south hemisphere is greater by a factor 1.12-1.21 than that of the north. In addition they found a southward shift of the heliospheric current sheet (HCS) of the order of 3-5 deg (see also Virtanen \& Mursula 2010). A previous limit of about $7 \mathrm{deg}$ was found by Mursula \& Hiltula (2004) based on heliospheric magnetic field observations at $1 \mathrm{AU}$. As of now, the actual values of the southward shift of the HCS remain not well known.

A possible model for explaining the $\mathrm{N}-\mathrm{S}$ asymmetry is to consider the global magnetic field as the contribution of several multipole components, and to ascribe the mismatch between the north and the south magnetic fields to the contribution of the quadrupole moment, as suggested by Bravo \& González-Esparza (2000) and Mursula \& Hiltula (2004). Indeed, Fig. 1 of Bravo \& González-Esparza (2000) and Bravo \& González-Esparza (2001), which compares the dipole, quadrupole, and esapole structures, shows how this asymmetry could be generated: if we look at the direction of the magnetic field for the dipole and the quadrupole during two consecutive solar minima, we note that they are opposite in the north pole and concordant in the south pole. The direction of the magnetic field of the esapole is always concordant with that of the dipole at the poles. This means that, because of the action of the quadrupole moment, the northern magnetic field is weakened, while the southern field is enhanced, causing the evident asymmetry and shift of the HCS in the southward direction.

Observations of non-radial white-light coronal streamers (Wang 1996) or the deflection of EUV polar plumes (de Patoul et al. 2013a,b) have facilitated the study of the possible configuration of the global and polar magnetic field, respectively. On the other hand, coronal jets are transient density enhancements of plasma that can highlight the local structure of the magnetic field. Indeed, hot jets at poles observed in EUV or X-rays are naturally explained as the result of the magnetic reconnection between emerging flux with the open magnetic field: the tension force of the disconnected field lines compresses the plasma and pushes it away along the magnetic field lines (Yokoyama \& Shibata 1995).

The aim of this work is to estimate the configuration of the solar magnetic field, and consequently the N-S asymmetry, starting from the latitudinal deflection of polar jets observed by the Solar TErrestrial RElations Observatory (STEREO) spacecraft. Section 2 presents the analysis of the deflection of the polar coronal jets from the catalogue of Nisticò et al. (2009), regarding jets observed from March 2007 to April 2008, which roughly corresponds to the solar minimum between the end of solar cycle 23 and the beginning of 24 . The analysis is performed in 2D and 3D space. Modeling and extrapolation of the coronal magnetic field structure is explained in Sect. 3, and conclusions are given in Sect. 4.

\section{Polar jet deflection measurements}

\section{1. $2 D$ analysis}

We investigate the influence of the large-scale coronal magnetic field on the motion of 79 jets through the corona observed with STEREO during March 2007-April 2008, which are catalogued in Nisticò et al. (2009). During this period, the angular separation between the two STEREO spacecraft increased from 2 to $\sim 48 \mathrm{deg}$. We exploited data from EUVI and COR1 instruments of the SECCHI package. Our method consists of calculating the position angle (PA), i.e. the angular displacement from the north axis to the jet position in counterclockwise direction, at two fixed distances from the solar centre: $1 R_{\odot}$, which corresponds to the solar limb, and $2 R_{\odot}$ (see Fig. 1). Since the EUVI images cover the full disk up to $1.7 R_{\odot}$, they are appropriate for measuring the PA at $1 R_{\odot}\left(\theta^{\mathrm{EUVI}}\right)$, which in most cases corresponds with the position of the jet footpoint. Images from COR1 have a field-of-view (FOV) within $1.4-4 R_{\odot}$, but the exact limits vary because of the offset of the occulter with respect to the Sun's centre. To clearly identify the jet in COR1, we took PAs at $2 R_{\odot}\left(\theta^{\mathrm{COR} 1}\right)$, somewhat above the boundary of the occulter from the Sun's centre. We prepped FITS file data within the SolarSoft (SSW) environment by secchi_prep.pro, which allows us to calibrate and apply corrections to the images, including rotation of the axis towards the solar north. Information about the Sun's centre in units of pixels are retrieved for EUVI from the keywords CRPIX1 and CRPIX2 of the header, and for COR1 we used the function wcs_get_pixel.pro ${ }^{1}$. The pixel size in arcsec is given by CDELT1 and CDELT2, and the solar radius by RSUN for both instruments. Circumferences defining the distance of 1 and $2 R_{\odot}$ from the centre are over-plotted in the images and the PA for each single jet is visually determined and measured in deg either from STEREO A or B, according to which spacecraft offers a better view in both instruments. We assume an error of $\Delta l=10$ pixels in the location of the jet (this apparently large error can include some other instrumental errors, jitter, etc.) with both instruments. This can be easily converted in terms of angular displacement, by considering the pixel size of each detector $\left(\Delta_{\text {pix }}\right)$ and the radial distance from the centre, which are not constant through the period of observations. Thus, a typical error in EUVI is $\Delta \theta^{\mathrm{EUVI}}=\Delta l \Delta_{\text {pix }} / r \approx 1 \mathrm{deg}$, (with $\Delta_{\text {pix }} \approx 1.6^{\prime \prime}$ and $r \approx 950^{\prime \prime}$ ), and in COR $1 \Delta \theta^{\mathrm{COR} 1} \approx 2 \mathrm{deg}$ (with $\Delta_{\text {pix }} \approx 7.5^{\prime \prime}$ and $\left.r \approx 1900^{\prime \prime}\right)$. The measurements of the PAs for the 79 jets from COR1 are plotted as a function of the corresponding PAs from EUVI in Fig. 2 for the north pole (left) and the south pole (right). The somewhat larger number of events in the north coronal hole with respect to the south (45 against 34, respectively) is due to the orbital features of STEREO, which allow for a better view of the northern region in the investigated time period (Nisticò et al. 2009). In addition, during the period of observations the Sun was at minimum. The jet angular positions, represented by circles, are fitted by a linear function separately for the north and south pole coronal hole (NPCH and $\mathrm{SPCH}$ ), according to the equation

$\theta^{\mathrm{COR} 1}=a \theta^{\mathrm{EUVI}}+b$,

where $a$ is the slope of the line and $b$ is the constant term of the linear fit. We performed linear fits with two different

1 The keywords CRPIX1 and CRPIX2 for COR1 return the centre of the occulter. Coordinates information can be retrieved with the World Coordinate System (WCS) routines in SSW starting from fitshead2wcs.pro. See http://hesperia.gsfc.nasa.gov/ssw/ gen/idl/wcs/wcs_tutorial.pdf 

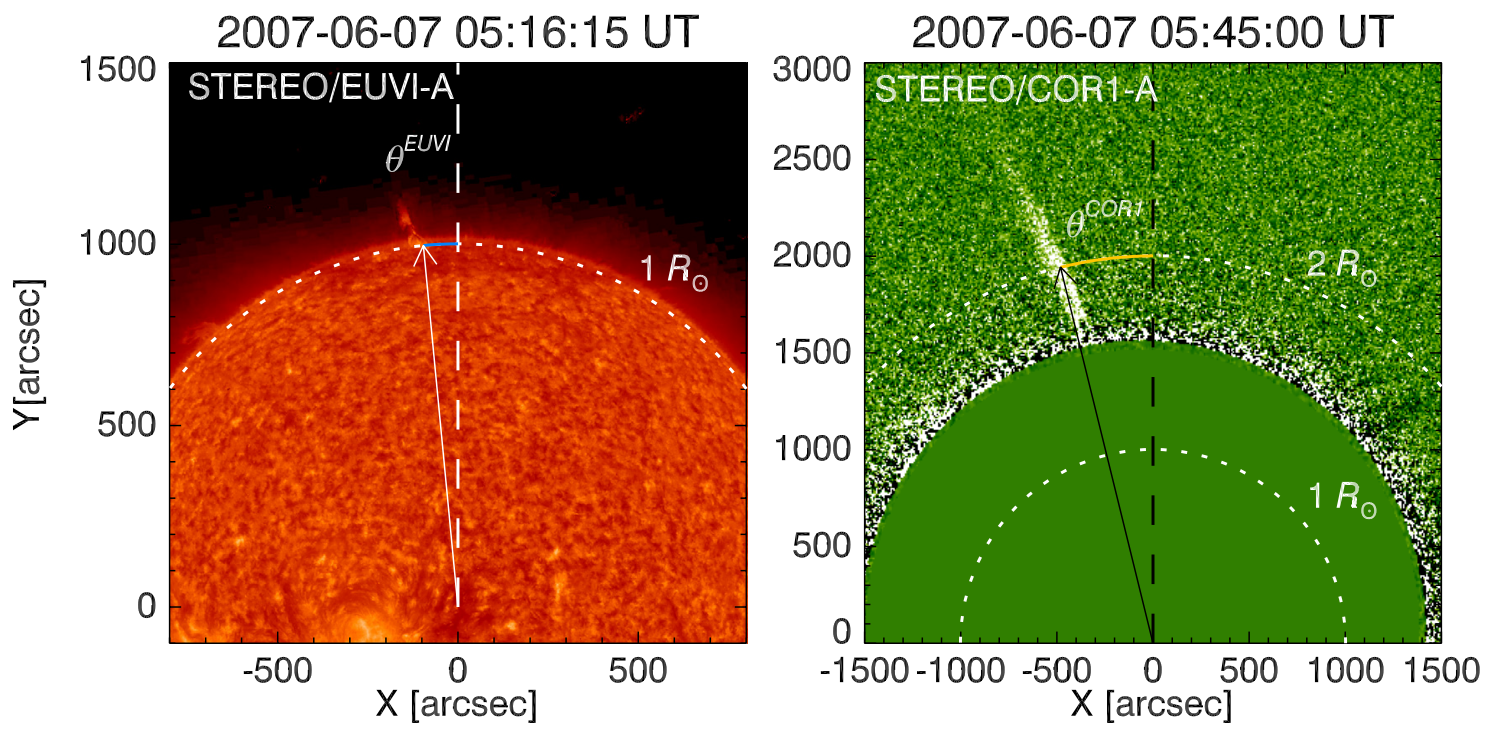

Fig. 1. Coronal jet in the NPCH observed by STEREO A at $304 \AA$ with EUVI (left) and in white-light with COR1 (right). PAs are measured from the north solar axis in the counterclockwise direction (see the reference frame overlapped on the images) at $1 R_{\odot}$ in the EUVI FOV and at $2 R_{\odot}$ in the COR1 FOV.

IDL routines: the first is a fit unweighted by measurement errors with linfit.pro, which performs regression of the data points along the vertical $y$-axis (dashed green lines in Fig. 2); and the second fit is performed with linfitex . pro of the MPFIT package (Markwardt 2009), which performs full-Cartesian regression by taking errors in both $x$ and $y$ variables into account (yellow line). Both the fits are almost coincident. The red line is a reference line corresponding to the case when jets had the same PAs at 1 and at $2 R_{\odot}$, implying radial propagation. Although some scatter of the data points is present, an overall trend is evident: jets with small PAs (if in the NPCH) or small displacements from the south polar axis (if in the SPCH) in the EUVI FOV show a small deviation in the COR1 FOV (they are near or on the red line, representing events that have the same PA when seen from both instruments); and jets having large PAs (if in the NPCH) or large displacements from the south polar axis (if in the SPCH) in the EUVI FOV (i.e. events that occur at lower latitudes) show greater deviation in COR 1 on average. This could be associated with the fact that the trajectory of the jets is not simply radial but bends towards the equator: the actual jet angle is the difference between the position vectors at the heights of 1 and $2 R_{\odot}$ (see the green dashed line that does not coincide with the red one). We can assume that jets propagate, on average, along the magnetic field lines, which are almost radial near the solar dipole axis, while those at lower latitudes deviate more markedly from the radial direction because of the dipolar structure. This is also consistent with the over-expansion towards low latitudes of the fast solar wind in polar coronal holes (e.g. Fisk 1996). The nonradial outward propagation of the jets is a property consistent with those of other coronal structures: e.g. coronal streamers and polar plumes extend non-radially (Wang 1996). In addition, nonradial seems to be the propagation of CMEs, as found in earlier studies (e.g. Cremades \& Bothmer 2004). It can be noticed that when going from EUVI to COR1, the changes in PA are larger in the NPCH than in the SPCH. More precisely, linear fits of the data points, with linfit.pro and linfitex.pro for the north and the south, give different values of the parameters $a$ and $b$ that are summarised in Table 1.

The parameter $a$, which represents the slope of the fitting line for the PAs, and hence a measure of the average bending of
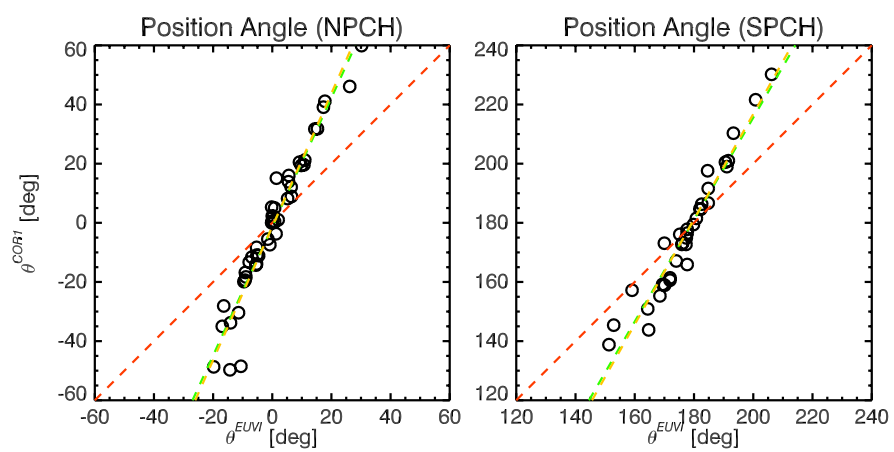

Fig. 2. COR1 PAs (vertical axis) vs. EUVI PAs (horizontal axis) for jets observed at the north (left) and at the south polar coronal hole (right). The red dashed line represents the bisector of the plane and ideally should trace equal PAs between the EUVI and COR1 FOV (radial propagation); the green dashed line fits the data points. Events rooted significantly away from the poles in EUV tend to have an even larger deviation in COR1.

jets, is indicated as $a_{\mathrm{N}}$ for the north (second column), and $a_{\mathrm{S}}$ for the south (fourth column) in Table 1. We also list the values of the parameter $b$, as $b_{\mathrm{N}}$ for the north (third column) and $b_{\mathrm{S}}$ for the south (fifth column), although they do not have a relevant role in the discussion. The bending is found to be on average greater in the north than in the south, giving an indication that jets are more deflected in the north than in the south $\left(a_{\mathrm{N}} / a_{\mathrm{S}}>1\right)$. The value of the quantity $\left(a_{\mathrm{N}}-a_{\mathrm{S}}\right) / a_{\mathrm{S}}$ shows that $a_{\mathrm{N}}$ is around $26 \%$ larger than $a_{\mathrm{S}}$. The square of the ratio of the coefficients, $\left(a_{\mathrm{N}} / a_{\mathrm{S}}\right)^{2}$, is also listed. The physical meaning of this quantity is discussed in the last section. This asymmetry is also evident in Fig. 3, where we plot the absolute value of the relative jet-bending from the solar axis $\left|\theta^{\mathrm{COR} 1}-\theta^{\mathrm{EUVI}}\right| /\left|\theta_{n}-\theta^{\mathrm{EUVI}}\right|$ as function of time for the north polar jets (black void triangles) and the south polar jets (red void squares). The parameter $\theta_{n}$, which represents the PA of the solar axis, is 0 deg at the north and $180 \mathrm{deg}$ at the south. We can infer that there is not a particular temporal dependence of the PA displacement from the EUVI to the COR1 FOV on time, as might be the case for Ulysses measurements during the fast 
Table 1. Values of the parameters for the linear fits of the EUV and COR1 PAs, latitudes, and longitudes for jets at the north and south poles.

\begin{tabular}{|c|c|c|c|c|c|c|}
\hline & $a_{\mathrm{N}}$ & $\begin{array}{c}b_{\mathrm{N}} \\
{[\mathrm{deg}]}\end{array}$ & $a_{\mathrm{S}}$ & $\begin{array}{c}b_{\mathrm{S}} \\
{[\mathrm{deg}]}\end{array}$ & $\left(a_{\mathrm{N}}-a_{\mathrm{S}}\right) / a_{\mathrm{S}}$ & $\left(a_{\mathrm{N}} / a_{\mathrm{S}}\right)^{2}$ \\
\hline PA & & & & & & \\
\hline LINFIT & $2.18 \pm 0.09$ & $-1.29 \pm 0.97$ & $1.72 \pm 0.09$ & $-129.20 \pm 15.44$ & $27 \%$ & $1.60 \pm 0.21$ \\
\hline LINFITEX & $2.25 \pm 0.04$ & $-1.31 \pm 0.45$ & $1.78 \pm 0.04$ & $-138.93 \pm 6.98$ & $26 \%$ & $1.61 \pm 0.09$ \\
\hline Latitude & & & & & & \\
\hline LINFIT & $1.77 \pm 0.36$ & $-72.33 \pm 28.59$ & $1.45 \pm 0.24$ & $47.64 \pm 18.26$ & $22 \%$ & $1.49 \pm 0.78$ \\
\hline LINFITEX & $2.31 \pm 0.06$ & $-115.51 \pm 4.97$ & $1.62 \pm 0.04$ & $56.74 \pm 3.06$ & $43 \%$ & $2.04 \pm 0.15$ \\
\hline Longitude & & & & & & \\
\hline LINFIT & $1.11 \pm 0.07$ & $-10.06 \pm 4.66$ & $1.00 \pm 0.02$ & $-1.53 \pm 2.42$ & - & - \\
\hline LINFITEX & $1.04 \pm 0.01$ & $2.08 \pm 0.51$ & $0.98 \pm 0.01$ & $0.81 \pm 0.77$ & - & - \\
\hline
\end{tabular}

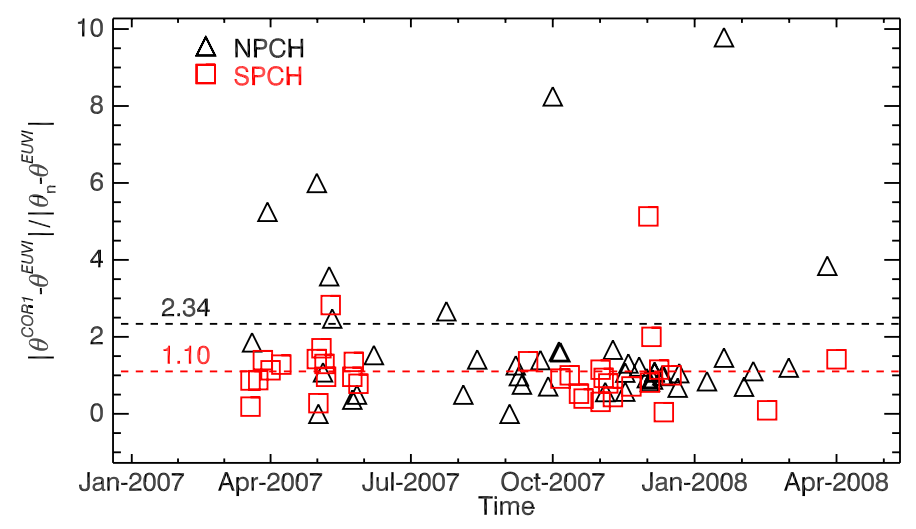

Fig. 3. Absolute angular displacement normalised to the EUVI PA relative to the solar axis as a function of time for jets occurred in the north (hollow black triangles) and in the south (hollow red squares). The quantity $\theta_{n}$ is 0 deg for events in the north and 180 deg for those in the south, in order to compare values between jets seen at the two poles. The dashed horizontal lines mark the average displacement: 2.34 deg for jets in the north and $1.10 \mathrm{deg}$ for those in the south.

latitude scans, and the average magnetic deflection is larger at the north pole than at the south pole.

The evidence that the observed jets are more deflected in the north pole than in the south pole could be associated with the different bending of the open magnetic field lines: in our case, jets occurring in the north are more deviated from their origin since the magnetic field lines are more curved than in the south during this period of observations.

\section{2. $3 D$ analysis}

The distinctive capability of STEREO is to perform 3D stereoscopic geometry or trajectory reconstruction of solar structures, such as loops (e.g. Nisticò et al. 2013), and CMEs (Bosman et al. 2012). Understanding the 3D evolution of jets through the corona as observed with the EUVI and COR1 instruments can provide additional information about their magnetic deflection. We measured the 3D position of jets in Stonyhurst longitude and latitude, at given radial distances of 1 and $2 R_{\odot}$. In practise, we used the routine scc_measure.pro available from the SSW package, which allows us to determine these quantities. We did this for a subset of the jets in the catalogue, i.e. for those events for which the determination of the $3 \mathrm{D}$ position is more reliable because of better viewing; this was possible for 38 events (20 at the north and 18 at the south pole, respectively). We identified the coordinates at $1 R_{\odot}$ by triangulating the base of the jet in EUVI images. For each event, we collected 10 measurements to take into account errors due to the triangulation process, and calculated the average values and standard deviations for the longitude and latitude. The same procedure has been performed for COR 1 to measure coordinates at $2 R_{\odot}$. In this case, collecting points at this fixed distance required more effort since there is not any reference that can help us to locate the jet (in the EUVI FOV, the footpoint of the jet or the limb is a good marker). The visibility of the jet, in both cases, has been eventually improved using difference images.

The results of the 3D measurements are shown in Fig. 4. The jet location is de-projected in polar plots, showing the Stonyhurst longitude (concentric circles) and latitude (radial lines) at $1 R_{\odot}$ as red dots, and at $2 R_{\odot}$ as green dots. A blue dashed line connects the locations of a jet, which is marked by a number according the catalogue from Nisticò et al. (2009). The radial lines and arcs centred on the dots are the error bars for the latitude and the longitude, respectively. These graphs provide an anticipation of what we can see with the Solar Orbiter, when it will be able to see the polar cap directly when orbiting out of the ecliptic plane. The first impression is the consistency of the measurements from EUVI and COR 1, which are taken independently, showing displacements towards low latitudes at higher distance from the Sun, and almost a radial trajectory (i.e. a small longitudinal shift). In a similar way as done for the PAs, we can fit the measurements between 1 and $2 R_{\odot}$ for the latitudes and longitudes, respectively, using (1). The scatter plots are shown in Fig. 5. The latitudes for both poles (top panels) are distributed far away from the bisector of the plane (red dashed line, which ideally should mark events with no change in latitude), and the linear fits (in green with linfit and yellow with linfitex) return a slope $a_{\mathrm{N}}=1.78-2.31$ for the north, and $a_{\mathrm{S}}=1.44-1.62$ for the south (see the second row group in Table 1). In this case, the quantity $\left(a_{\mathrm{N}}-a_{\mathrm{S}}\right) / a_{\mathrm{S}}$ shows that $a_{\mathrm{N}}$ is around 22-43\% larger than $a_{\mathrm{S}}$, which is almost consistent with the results obtained from the PA measurements. The ratio $\left(a_{\mathrm{N}} / a_{\mathrm{S}}\right)^{2}$ is around 1.5-2.0. On the contrary, the longitudes (third row group in Table 1) are very close to the bisector (slopes are 1.11-1.04 and 1.00-0.98 for the north and south jets, respectively), suggesting that the studied jets did not exhibit a significant shift in the azimuthal direction.

\section{A model for the coronal magnetic field}

From the results shown in the previous section, a natural question arises: "Is the different deflection of jets, in the north and in the south poles, an indication of a magnetic N-S asymmetry?". There are several extrapolation methods for estimating the magnetic configuration of the solar corona. For details see Altschuler \& Newkirk (1969), Kivelson \& Russell (1995), and Aschwanden (2005, Chap. 5). In the potential field (i.e. 

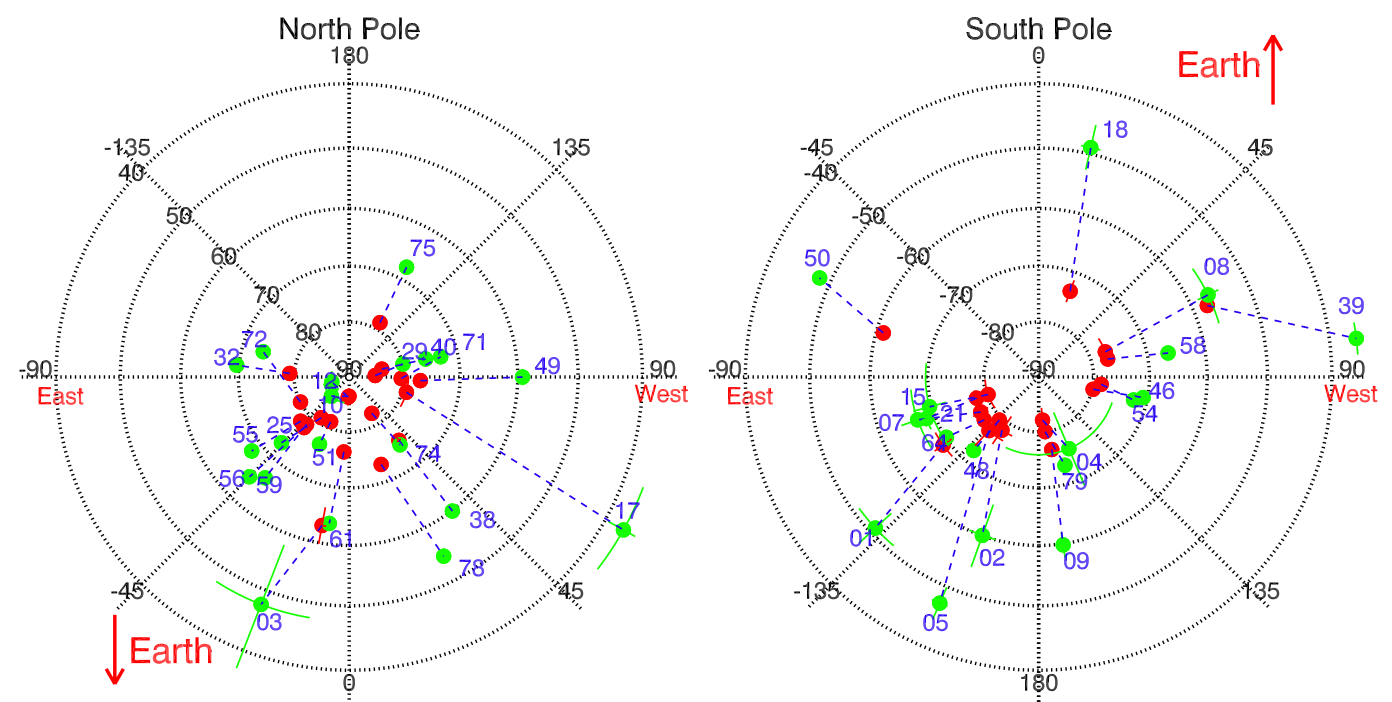

Fig. 4. Polar plots of the 3D positions of jets seen at the north (left) and south poles (right). The circles represent the heliographic longitudes and the radial lines the latitude meridians measured in deg. The positions of jets at $1 R_{\odot}$ are in red, at $2 R_{\odot}$ in green, respectively. The Sun-Earth direction is given by a red arrow in both plots.
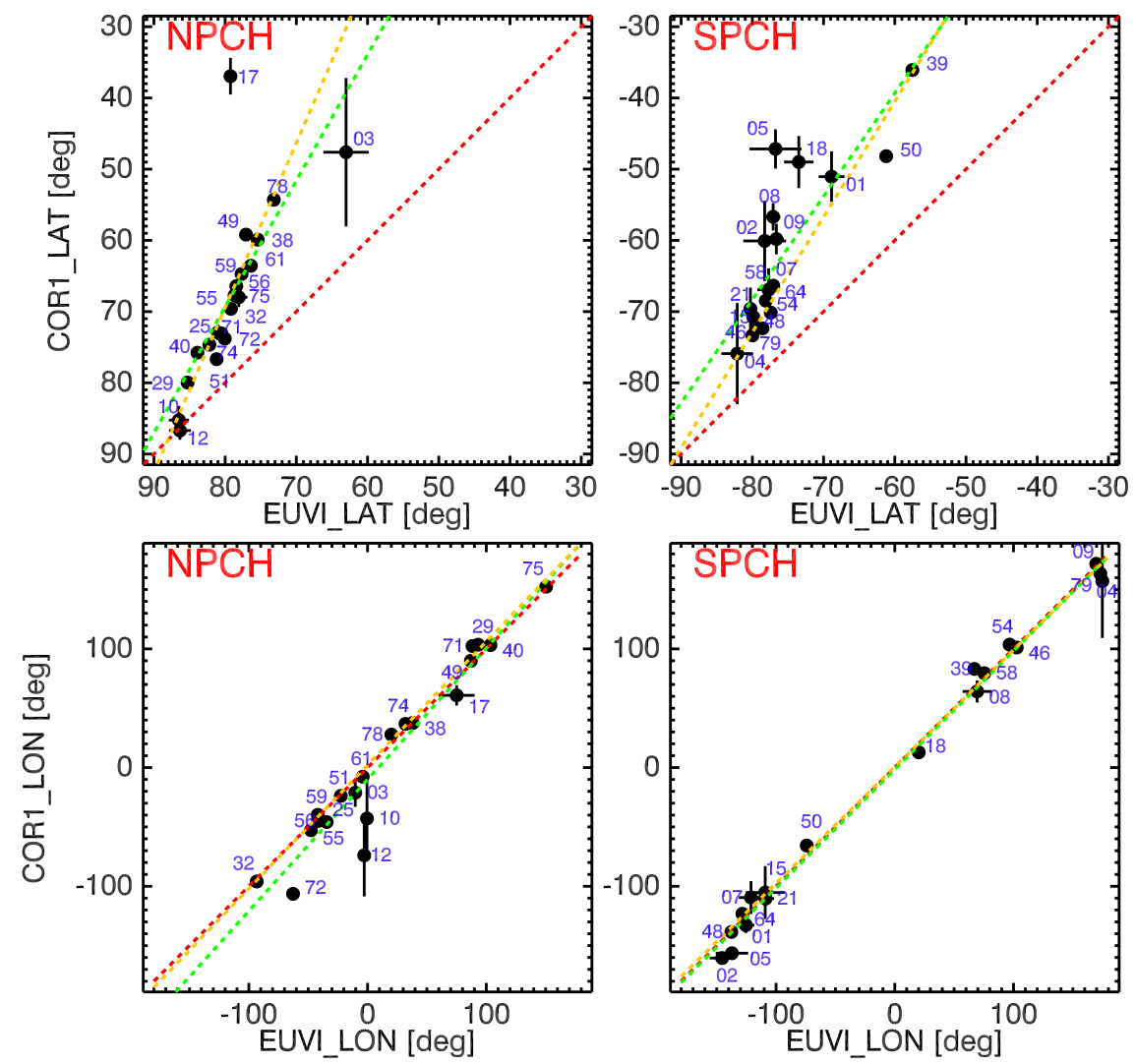

Fig. 5. Top: scatter plots of the latitudes measured with EUVI at $1 R_{\odot}$, and COR 1 at $2 R_{\odot}$ for the north (left) and south (right) polar jets. Linear fits of the points are showing green and yellow dashed lines, while the red line is the bisector of the plane. Bottom: as the previous graphs, scatter plots of the longitudes are shown. The numbers in blue identify the events in the catalogue of Nisticò et al. (2009).

current-free) approximation, the magnetic field can be obtained as the gradient of a scalar potential, $\boldsymbol{B}=-\nabla \Psi$, and the scalar potential function can be expressed in spherical coordinates as an expansion in terms of the Legendre polynomials $P_{l}^{m}(\cos \theta)$, i.e.

$$
\begin{aligned}
\Psi(r, \theta, \phi)= & R_{\odot} \sum_{l=1}^{N} \sum_{m=0}^{l} f_{l}(r) P_{l}^{m}(\cos \theta) \\
& \times\left[g_{l}^{m} \cos (m \phi)+h_{l}^{m} \sin (m \phi)\right] .
\end{aligned}
$$

The indices $l$ and $m$ are integer numbers: $l$ is strictly positive and defines the number of axes of symmetry of the field, while $m$ can assume $2 l+1$ values $(-l, \ldots,-1,0,1, \ldots, l)$ and defines the orientation of these axes in spherical geometry. The function

$f_{l}(r)=\frac{\left(r_{w} / r\right)^{l+1}-\left(r / r_{w}\right)^{l}}{\left(r_{w} / R_{\odot}\right)^{l+1}-\left(R_{\odot} / r_{w}\right)^{l}}$

fixes the position of the solar wind source surface at $r_{w}$ solar radii; beyond $r_{w}$ the magnetic field lines are purely radial, reproducing the configuration of the magnetic field in the solar wind (Aschwanden 2005). Thus, Eqs. (2), (3) must be used only for $R_{\odot}<r<r_{w}$. 
The components of the magnetic field can be found as the derivative of the scalar potential $\Psi$. We can further simplify the expressions of the magnetic field components, if we assume axial symmetry. This assumption is supported by the 3D analysis of the jet positions, showing negligible shift in the longitudinal or azimuthal direction: then the component $B_{\phi}$ is null. Of course, this assumption implies that we are neglecting other effects like the possible magnetic dipole tilt. Axial symmetry is enforced by setting $m=0$. Indeed, near the poles $(\theta \simeq 0 \mathrm{deg}, 180 \mathrm{deg})$, all associated Legendre polynomials $P_{l}^{m} \propto \sin ^{m} \theta \rightarrow 0$, except for $m=0$.

The expansion now only depends on the index $l$. If we truncate the series at $l=3$, we have three contributions that give the dipole $(l=1)$, the quadrupole $(l=2)$, and the esapole $(l=3)$ terms, with the corresponding moments (or harmonic coefficients) $g_{1}, g_{2}, g_{3}$ (we dropped the superscript $m$ in the coefficients since it is always 0 ).

After some algebra, we find the following magnetic field components due to the dipole:

$B_{r}^{(1)}(r, \theta)=\left(\frac{R_{\odot}}{r}\right)^{3}\left(\frac{2 r_{w}^{3}+r^{3}}{r_{w}^{3}-R_{\odot}^{3}}\right) g_{1} \cos \theta$,
$B_{\theta}^{(1)}(r, \theta)=\left(\frac{R_{\odot}}{r}\right)^{3}\left(\frac{r_{w}^{3}-r^{3}}{r_{w}^{3}-R_{\odot}^{3}}\right) g_{1} \sin \theta ;$

the components due to the quadrupole:

$$
\begin{aligned}
& B_{r}^{(2)}(r, \theta)=\frac{1}{2}\left(\frac{R_{\odot}}{r}\right)^{4}\left(\frac{3 r_{w}^{5}+2 r^{5}}{r_{w}^{5}-R_{\odot}^{5}}\right) g_{2}\left(3 \cos ^{2} \theta-1\right), \\
& B_{\theta}^{(2)}(r, \theta)=3\left(\frac{R_{\odot}}{r}\right)^{4}\left(\frac{r_{w}^{5}-r^{5}}{r_{w}^{5}-R_{\odot}^{5}}\right) g_{2} \cos \theta \sin \theta ;
\end{aligned}
$$

and the components due to the esapole:

$$
\begin{aligned}
& B_{r}^{(3)}(r, \theta)=\frac{1}{2}\left(\frac{R_{\odot}}{r}\right)^{5}\left(\frac{4 r_{w}^{7}+3 r^{7}}{r_{w}^{7}-R_{\odot}^{7}}\right) g_{3}\left(5 \cos ^{3} \theta-3 \cos \theta\right), \\
& B_{\theta}^{(3)}(r, \theta)=\frac{1}{2}\left(\frac{R_{\odot}}{r}\right)^{5}\left(\frac{r_{w}^{7}-r^{7}}{r_{w}^{7}-R_{\odot}^{7}}\right) g_{3}\left(15 \cos ^{2} \theta \sin \theta-3 \sin \theta\right) .
\end{aligned}
$$

In the limit of $r_{w} \rightarrow \infty$, we have the classical expressions for the dipole, the quadrupole, and the esapole in free space. The resulting magnetic field can be written as the sum of the dipole, quadrupole, and esapole contributions, i.e.

$B_{r}(r, \theta)=B_{r}^{(1)}+B_{r}^{(2)}+B_{r}^{(3)}$,
$B_{\theta}(r, \theta)=B_{\theta}^{(1)}+B_{\theta}^{(2)}+B_{\theta}^{(3)}$.

The magnetic field lines can be obtained by integrating, with a Runge-Kutta scheme of the 4th order for example, the two first order differential equations, i.e.

$$
\left\{\begin{array}{l}
\mathrm{d} r / \mathrm{d} s=B_{r} / B \\
\mathrm{~d} \theta / \mathrm{d} s=B_{\theta} / r B
\end{array}\right. \text {, }
$$

where $s$ is the distance along the field line. This model is then used to find a relationship between the coefficients $a$ and the normalised multipole coefficients of the reduced field model.

\subsection{Magnetic moments from the Wilcox Solar Observatory}

Given the model for the coronal magnetic field with the assumption of axial symmetry and the truncation to the dipole, quadrupole and esapole moments, we can search which values of the magnetic moments $g_{1}, g_{2}, g_{3}$, are suitable for better describing the jet magnetic deflections. For comparison, these coefficients are calculated from magnetograms data, provided and published by the Wilcox Solar Observatory (WSO) ${ }^{2}$. To infer values of these coefficients from magnetograms, two kinds of hypotheses are made regarding the inner boundary conditions in the photosphere (Wang \& Sheeley 1992). Indeed, from magnetograms we can measure the line-of-sight (LOS) component of the photospheric field at a given latitude $\alpha$ on the solar disk. The "classic" model takes the projection of the LOS photospheric field along the radial and latitudinal component into account $\left(B_{r}=B_{\mathrm{LOS}} \cos \alpha, B_{\theta}=B_{\mathrm{LOS}} \sin \alpha\right)$; conversely, the "radial" model assumes that the photospheric field is totally radial $\left(B_{r}=\right.$ $\left.B_{\mathrm{LOS}} / \cos \alpha, B_{\theta}=0\right)$. Since the magnetic field is non-potential and nearly radial at the photosphere (Wang \& Sheeley 1992), the radial model is considered to be a better approach.

The top panels of Fig. 6 show the temporal evolution of the coefficients for the classic and the radial model, as calculated by WSO. The bottom plots are the temporal evolution of the ratios $g_{2}^{W} / g_{1}^{W}$ and $g_{3}^{W} / g_{1}^{W}$ (where the superscript $W$ signifies $g$ coefficients calculated by WSO). The region bounded by the dashed lines is the temporal window in which our jets are observed.

We can see that the harmonic coefficients evolve in time, and that the quadrupole moment is lower in magnitude than the other moments, and exhibits an oscillatory behaviour, especially in the radial model, with alternating sign and with a period of approximately two years, indicating a possible association with biennal oscillations (Vecchio \& Carbone 2008; Vecchio et al. 2012; Bazilevskaya et al. 2014). In contrast, the dipole and esapole moments show a constant sign. Below, we try to calculate these coefficients in a different way and to compare them with those obtained from WSO. Further, we find the associated coronal magnetic field structure and the position of the HCS projected on the solar surface.

\subsection{Fitting the magnetic field model to the polar jets PAs}

Here, we estimate the best values of the coefficients $g_{2}$ and $g_{3}$ that fit our jet observations and measurements of PA, in terms of the dipole moment (which is used as a normalisation factor). For several values of $\hat{g}_{2}=g_{2} / g_{1}$ and $\hat{g}_{3}=g_{3} / g_{1}$ coefficients, we integrate numerically the equations (12) from the base of the jets, i.e. the PA as measured in the EUVI FOV, up to $2 R_{\odot}$. This yields the difference between the final PA from the numerical integration, $\theta^{\mathrm{COR} 1_{\text {Mod }}}$, which is a function of $\hat{g}_{2}$ and $\hat{g}_{3}$, and that measured in the COR $1 \mathrm{FOV}, \theta^{\mathrm{COR} 1_{\mathrm{Obs}}}$. Then we calculate the standard deviation $\sigma\left(\hat{g}_{2}, \hat{g}_{3}\right)$ as

$$
\sigma\left(\hat{g}_{2}, \hat{g}_{3}\right)=\sqrt{\frac{\sum_{i=1}^{N}\left[\theta_{i}^{\mathrm{COR} 1_{\mathrm{Mod}}}\left(\hat{g}_{2}, \hat{g}_{3}\right)-\theta_{i}^{\mathrm{COR} 1_{\mathrm{Obs}}}\right]^{2}}{N-1}},
$$

with $N$ the total number of magnetic field lines successfully integrated from the jet base at $1 R_{\odot}$ up to $2 R_{\odot}$. The number $N$ is not necessarily 79 but can be less, depending on the values of the magnetic moments since the magnetic field lines can be closed without reaching $2 R_{\odot}$. Figure 7 shows for example some magnetic field lines integrated for a few jets with given values of the coefficients $\hat{g}_{2}$ and $\hat{g}_{3}$. In some events, a good agreement between the final position from the integration and the observed PA at $2 R_{\odot}$ is found and some others show a considerable gap,

\footnotetext{
2 See the website http://wso.stanford.edu/
} 

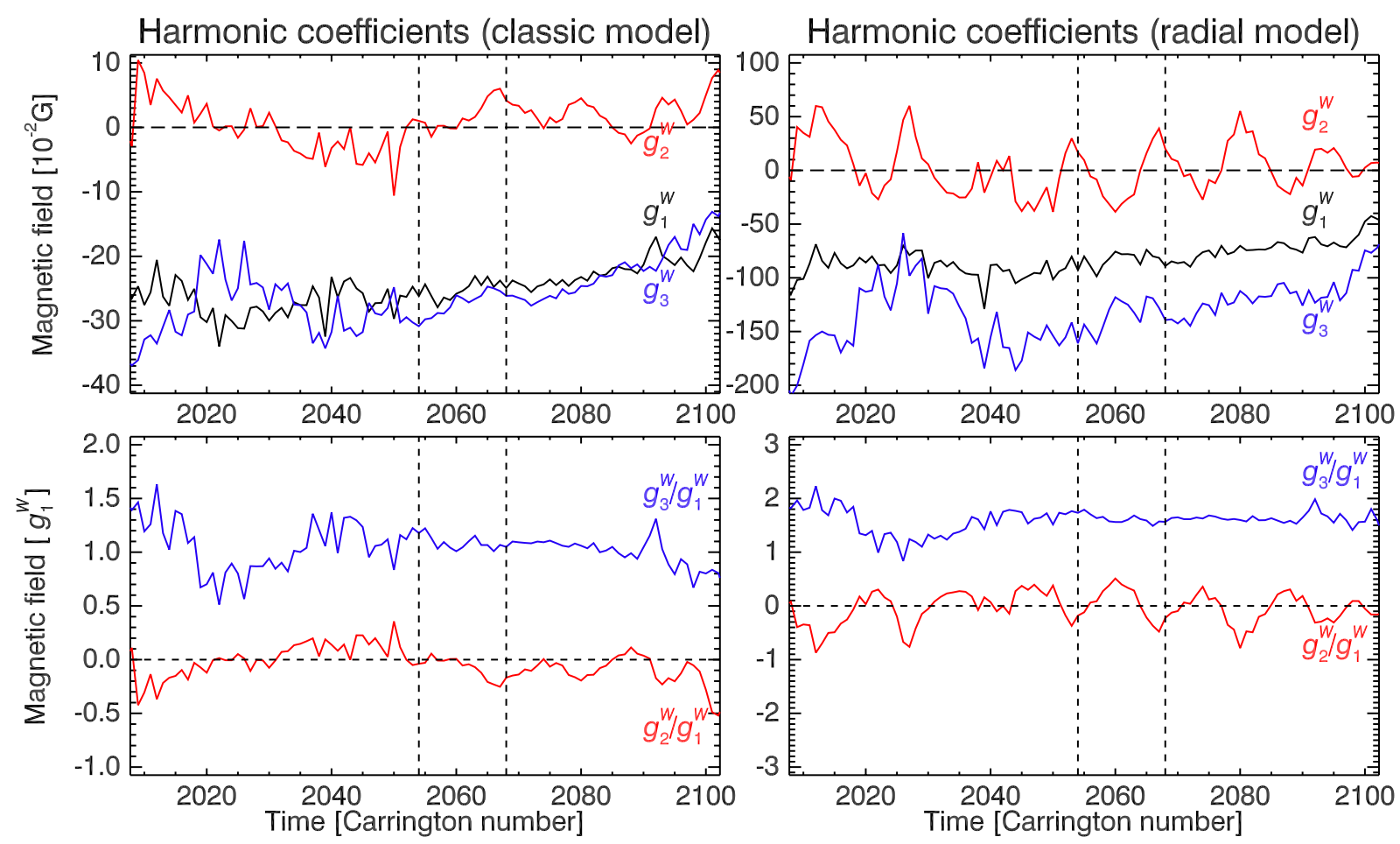

From 2003-11-19 to 2010-08-09

Fig. 6. Top: time evolution of the harmonic coefficients $g_{1}^{W}, g_{2}^{W}, g_{3}^{W}$, as calculated by the Wilcox Solar Observatory in the classic (left) and radial (right) model. Bottom: time evolution of the quadrupole and esapole moments normalised to the dipole component. Note the periodic change of the quadrupole over a scale of 20 Carrington rotation $(\sim 2 \mathrm{yr})$ in the radial model. The vertical dashed lines enclose the period of our observations.

whilst for one event, marked by a purple dashed line, the integrated line results to be closed and does not reach $2 R_{\odot}$. The coefficient $\hat{g}_{2}$ spans from -3 to 3 and $\hat{g}_{3}$ from 0 to 3 , both in steps of 0.1 . We give the results of $\sigma\left(\hat{g}_{2}, \hat{g}_{3}\right)$ as $2 \mathrm{D}$ contour maps representing the value of the standard deviation as a function of the quadrupole (vertical axis) and esapole (horizontal axis) moments, using different models of the magnetic field, i.e. classic and radial, and different distances of the solar source surface $r_{w}=2.5$ and $3.25 R_{\odot}$ (Altschuler \& Newkirk 1969; Hoeksema $1995)$. The grid in the maps is determined by the varying values of $\hat{g}_{2}$ and $\hat{g}_{3}$, with a resolution of 0.1 for both parameters, as used in the numerical model.

The top panels of Fig. 8 show the standard deviation maps for the classic (left) and radial (right) model considering the solar source surface at $2.5 R_{\odot}$; the bottom row gives the same results for $r_{w}=3.25 R_{\odot}$. The colourbar provides the variation range of $\sigma$ : from 4 (black) to $16 \mathrm{deg}$ (red). The purpose of these maps is to give us an indication of which values of $\hat{g}_{2}$ and $\hat{g}_{3}$ minimise the standard deviation (dark regions in the maps), and better fit the deflections of jets. In addition, they are overlaid by some coloured lines that enclose portions of the maps characterised by the same percentage of jets, hence magnetic field lines, successfully integrated. Indeed, if a certain value of $\sigma$ is obtained for different values of the magnetic moments, the number of integrated lines $N$ provides a further constrain to discriminate which values of $\hat{g}_{2}$ and $\hat{g}_{3}$ are more reliable: the higher $N$, the better the adaptation of the magnetic field model to our jet observations. For this reason, we can exclude the minimum in $\sigma$ found for $\hat{g}_{2} \sim-2.5$ and $\hat{g}_{3} \sim 1.8$, since it is obtained for as few as almost $50 \%$ of the observed jets.

We do not obtain specific and exclusive values of magnetic moments that fit our observations. The shape of the low- $\sigma$ is

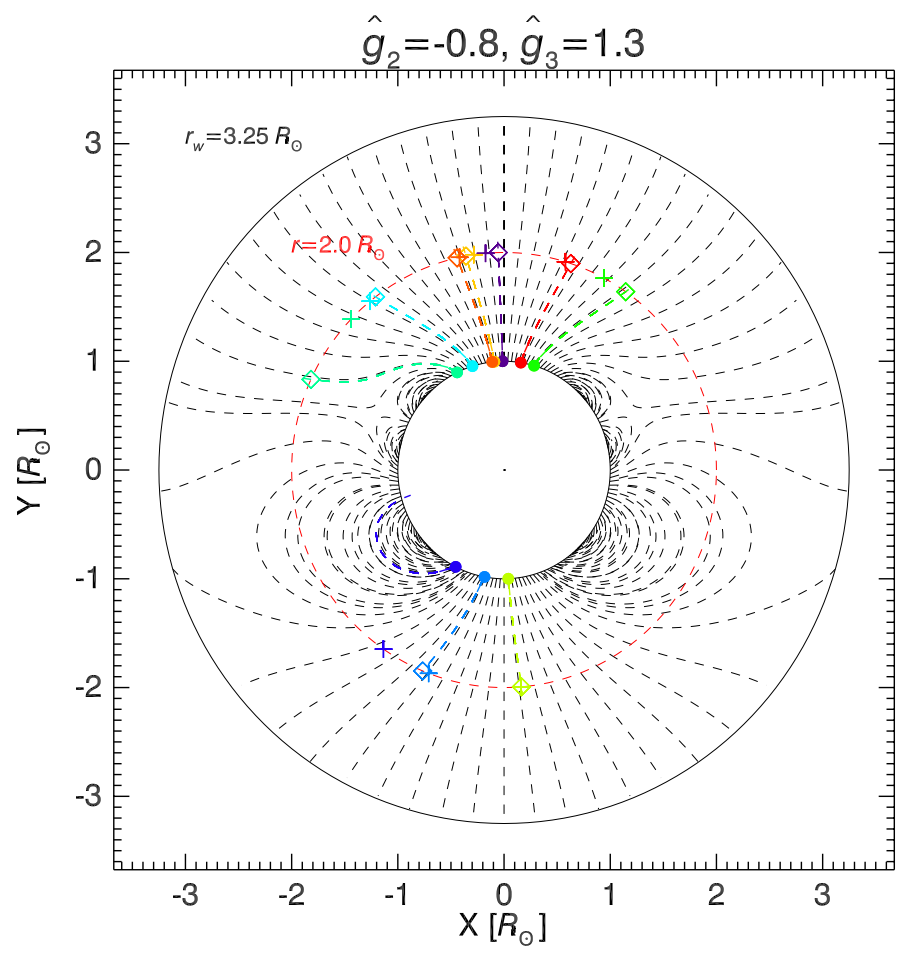

Fig. 7. Example of magnetic field lines integration for some jets with moments $\hat{g}_{2}=-0.8$ and $\hat{g}_{3}=1.3$. The starting points of the integration are at $1 R_{\odot}$ from EUVI observations and represented as coloured dots. The integrations are made up to $2 R_{\odot}$ and the results (void squares) are compared with the corresponding jet positions as observed with COR1 (plus signs). For some events, especially those at lower latitudes, the integration can result in a closed line (purple dashed line). 

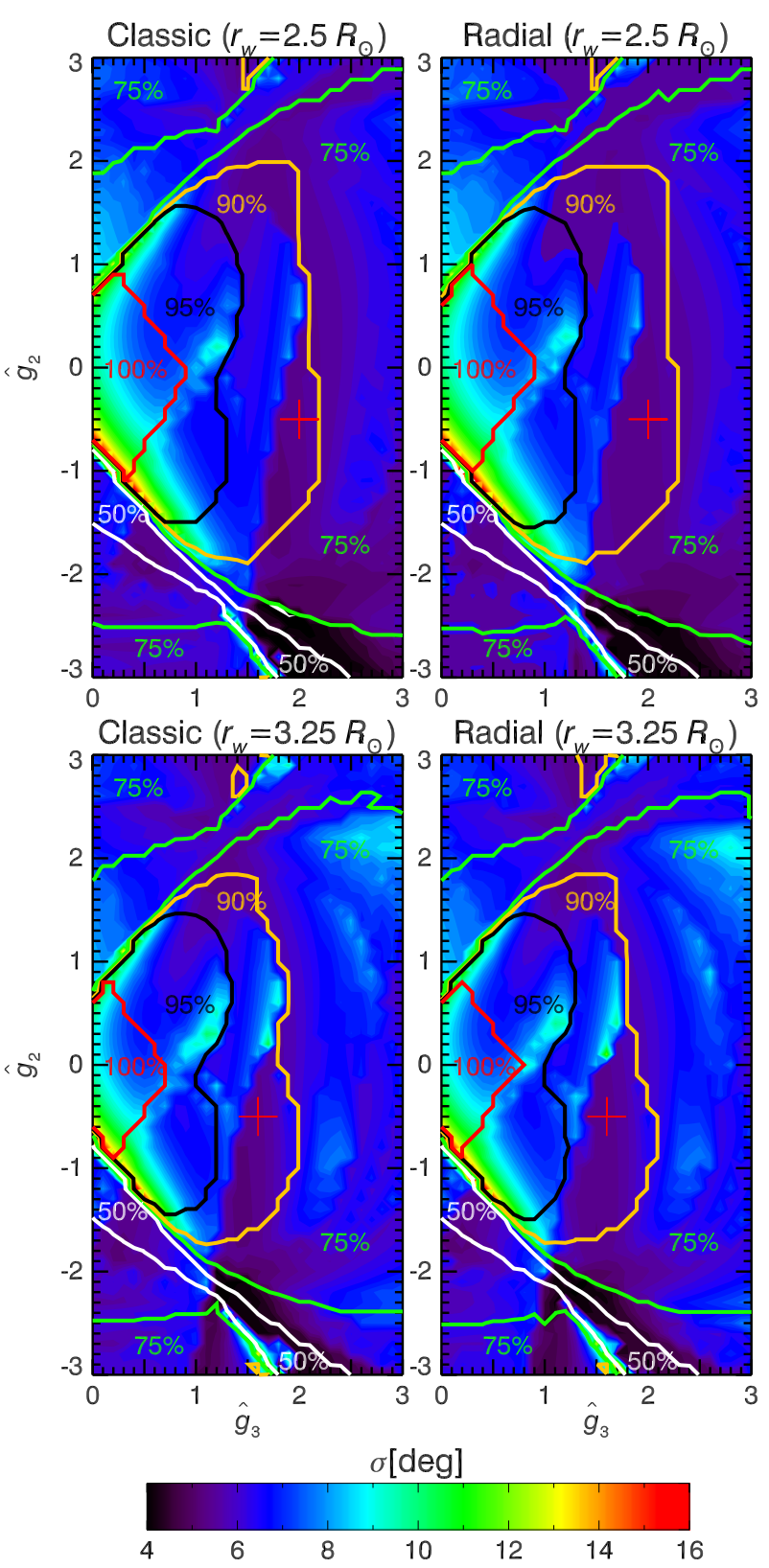

Fig. 8. Contour maps representing the standard deviation $(\sigma)$ from (13) for different values of $r_{w}$ (top: $2.5 R_{\odot}$, bottom: $3.25 R_{\odot}$ ), for the classic (left) and radial (right) model, as a function of $\hat{g}_{2}$ (vertical axis) and $\hat{g}_{3}$ (horizontal axis) coefficients. The colourbar defines the variation range of $\sigma$. In addition, coloured lines enclose regions with different percentage $(100,95,90, \ldots)$ of jets, hence magnetic field lines, which are successfully integrated. The red crosses locate the chosen minimum in a low- $\sigma$ region with at least $90 \%$ of jets integrated. This minimum is found for $\left\langle\hat{g}_{2}\right\rangle=-0.5$, and $\left\langle\hat{g}_{3}\right\rangle=1.6$ (bottom panels) 2.0 (top panels).

almost similar in all cases and appears to be more sensitive to the esapole moment, since it is more narrowed for some values of $\hat{g}_{3}$, and spread for several values of $\hat{g}_{2}$, but a patch with $\sigma \leq 6 \mathrm{deg}$ and in the limit of $N=90 \%$ is found for negative values of the quadrupole, in agreement with the values from WSO. This is marked by red crosses in the maps at values of $\left\langle\hat{g}_{2}\right\rangle \sim-0.5$ and $\left\langle\hat{g}_{3}\right\rangle \sim 1.6-2.0$.

We can now infer the structure of the coronal magnetic field by computing Eqs. ((4)-(9)) with the obtained values of $\left\langle\hat{g}_{2}\right\rangle$ and $\left\langle\hat{g}_{3}\right\rangle$ and plotting the magnetic field lines. This is shown in

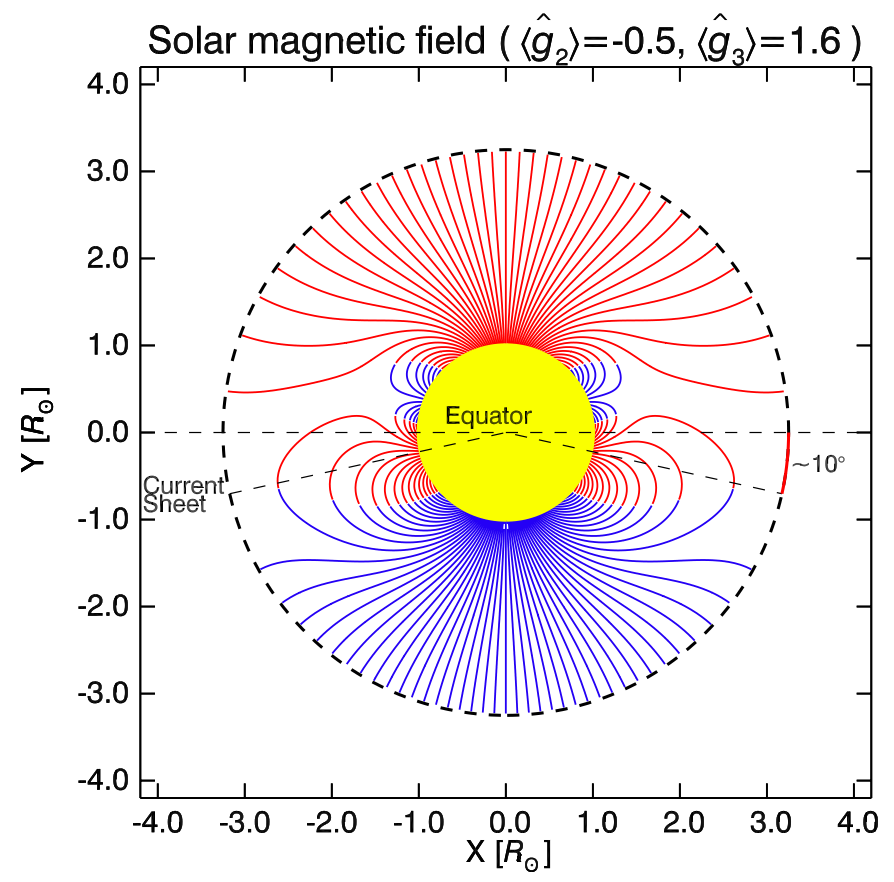

Fig. 9. Structure of the coronal magnetic field lines with $\left\langle\hat{g}_{2}\right\rangle=-0.5$ and $\left\langle\hat{g}_{3}\right\rangle=1.6$, and the corresponding HCS position projected on the source surface at $3.25 R_{\odot}$ for the interest period of observations between March 2007 and April 2008. Inwards magnetic field lines are plotted in red, outwards in blue.

Fig. 9: the HCS in the considered period of observations results to be coned southward, forming an angle of about $10 \mathrm{deg}$, which is broadly consistent, although somewhat larger, with some estimates found in the literature ranging between 3 and $10 \mathrm{deg}$ (Simpson et al. 1996; Heber et al. 1996; Mursula \& Hiltula 2004; Erdős \& Balogh 2010). On the other hand, the HCS is not a stationary feature and the tilt is subject to a change over the time because of the evolution of the magnetic field structure. For the period under examination, the average values of the quadrupole and esapole components, as determined by WSO, are of the order of $g_{2}^{W} / g_{1}^{W} \sim-0.1,0.05$ and $g_{3}^{W} / g_{1}^{W} \sim 1.0,1.6$ for the classic and radial model, respectively. In addition the quadrupole component shows a large variability, assuming positive and negative values with peaks at $\pm 0.5 g_{1}^{W}$ in the case of the radial approximation.

\section{Discussion and conclusions}

In this work, we used polar corona jets as a probe for understanding the magnetic field structure of the solar corona during a solar minimum. Since the corona is an environment with a plasma- $\beta$ parameter less than 1 , we assumed that jets on average propagate along the magnetic field lines. For simplicity, we also assumed that the large-scale solar magnetic field during a solar minimum is axisymmetric. We measured the PA of 79 polar jets at $1 R_{\odot}$ in the EUVI FOV, and at $2 R_{\odot}$ in the COR 1 FOV, and analysed the deflection of these jets. The analysis was also carried out in $3 \mathrm{D}$ space. We found that the jets are deflected towards low latitudes, in agreement with the fact that the magnetic field lines are bent towards the equator, and this deflection is more pronounced at the north pole than at the south pole. This N-S asymmetry has been found in other data sets, starting from photospheric magnetic field measurements (Hoeksema 1995), global distribution of the solar wind speed (Tokumaru et al. 2015), analysis 
of latitudinal gradient in solar energetic particles (Simpson et al. 1996; Heber et al. 1996), and also magnetic field measurements in the interplanetary medium by the Ulysses spacecraft (Erdôs $\&$ Balogh 2010). This asymmetry can be modelled in terms of multipole components of the global magnetic field (Bravo \& González-Esparza 2000; Mursula \& Hiltula 2004): during solar minima the quadrupole moment tends to influence the total magnetic field, weakening it in one pole and enhancing it in the opposite pole. We have estimated the contribution of the quadrupole moment, starting from the jet PA data, and compared our results with those of WSO. We modelled the coronal magnetic field as the sum of the dipole, quadrupole, and esapole moments, starting from the expression of a scalar potential function $\Psi$ in terms of spherical harmonic expansion. We traced the magnetic field lines from the footpoint of the jets (at $1 R_{\odot}$ ) up to $2 R_{\odot}$ for different values of the magnetic moments. We obtained the harmonic coefficients $\hat{g}_{2}$ and $\hat{g}_{3}$, normalised to the dipole, which minimise the standard deviation of the position angle differences between the numerical simulation results and the observations. We obtained as reliable values $\left\langle\hat{g}_{2}\right\rangle=-0.5$ and $\left\langle\hat{g}_{3}\right\rangle=1.6$. From these estimates, we were able to compute the structure of the magnetic field lines (Fig. 9) in which the HCS shows an offset of about $10 \mathrm{deg}$, a value consistent with the results obtained by Mursula \& Hiltula (2004), but somewhat larger than that found by Erdős \& Balogh (2010), whose observations however correspond in part to different periods and are taken at much larger distances from the Sun, around 1.4 AU during the fast latitude scans of Ulysses.

In our analysis, we show that the slopes of the linear fits for the PAs and the latitudes (from 3D measurements) are different between the two poles. The angular coefficients $a_{\mathrm{N}}$ and $a_{\mathrm{S}}$ of the fits can be immediately related to the ratio of the magnetic field values $B_{\mathrm{S}} / B_{\mathrm{N}}=1.12-1.21$, which is reported in Table 1 from Erdős \& Balogh (2010), according to the following geometric interpretation in terms of conservation of magnetic fluxes through the Sun's poles. Consider the sketch of the Sun in Fig. 10, with the polar caps marked by dashed lines at the distance of $1 R_{\odot}$, which can essentially represent areas embedded in open field regions, such as the polar coronal holes. We can express the magnetic fluxes $\Phi$ approximately as the product of the area $A$ of the polar cap with an average polar magnetic field $B$. The surface of a spherical cap depends on the half opening angle $\theta$ as $A=2 \pi R_{\odot}^{2}(1-\cos \theta)$, which we assume to be equal for both poles at $1 R_{\odot}$, for example. The angle $\theta$ is analogous to the PA in our measurements. Hence, because of the asymmetry, the flux $\Phi_{\mathrm{S}}$ in the south pole is larger than $\Phi_{\mathrm{N}}$ in the north. Thus, we find at the distance of $1 R_{\odot}$,

$A_{\mathrm{N}}\left(R_{\odot}\right)=A_{\mathrm{S}}\left(R_{\odot}\right) \Rightarrow \frac{\Phi_{\mathrm{N}}\left(R_{\odot}\right)}{\Phi_{\mathrm{S}}\left(R_{\odot}\right)}=\frac{B_{\mathrm{N}}\left(R_{\odot}\right)}{B_{\mathrm{S}}\left(R_{\odot}\right)}$.

Moving away of the Sun, the magnetic field diminishes with the distance $r$ and the area of the projected polar cap must increase because of the flux conservation $\left(\Phi_{i}\left(R_{\odot}\right)=\Phi_{i}(r)\right.$ with $i=\mathrm{N}, \mathrm{S})$. We can consider the variation of the polar magnetic field normalised to its values at the distance of $1 R_{\odot}$ as $B_{i}(r)=\hat{B}_{i}(r) B_{i}\left(R_{\odot}\right)$, and the expansion of the polar caps with the distance can be addressed in terms of the variation of the opening angle $\theta$ of a factor $a_{i}$, which is different between the two hemispheres (and also depends on the radial distance). Thus, at a certain distance $r$, which is taken to be $R_{\odot}<r \leq r_{w}$ in the PFSS model, the polar cap areas can be expressed as

$A_{i}(r)=2 \pi r^{2}\left[1-\cos \left(a_{i}(r) \theta\right)\right]$

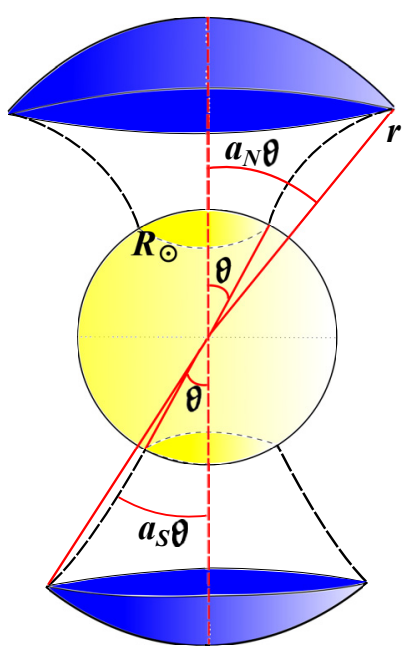

Fig. 10. Sketch representing the N-S asymmetry of the solar magnetic field in terms of magnetic fluxes through the polar caps. The polar caps on the solar disk at the distance of $1 R_{\odot}$ ideally represent regions in polar coronal holes with equal area, which depend upon the half-opening angle $\theta$. Because of the asymmetric over-expansion of the magnetic field lines between the two hemispheres (represented by the curved dashed lines departing from the solar disk), the angle $\theta$ at a given distance $r$ increases by a factor $a_{\mathrm{N}}$ in the north and $a_{\mathrm{S}}$ in the south, with $a_{\mathrm{N}}>a_{\mathrm{S}}$. The resulting size of the northern polar cap is larger than the southern cap.

with $i=\mathrm{N}, \mathrm{S}$. A second order approximation for the cosine function gives $\cos (a \theta) \approx 1-\frac{1}{2} a^{2} \theta^{2}$, and, finally, the area can be expressed as $A_{i}(r)=\pi r^{2}\left(a_{i}^{2} \theta^{2}\right)$. By considering the ratio of the magnetic fluxes between the north and south at $r$, we find,

$\frac{\Phi_{\mathrm{N}}(r)}{\Phi_{\mathrm{S}}(r)}=\frac{B_{\mathrm{N}}(r) A_{\mathrm{N}}(r)}{B_{\mathrm{S}}(r) A_{\mathrm{S}}(r)} \approx \frac{\hat{B}_{\mathrm{N}}(r)}{\hat{B}_{\mathrm{S}}(r)} \frac{B_{\mathrm{N}}\left(R_{\odot}\right)}{B_{\mathrm{S}}\left(R_{\odot}\right)}\left(\frac{a_{\mathrm{N}}(r)}{a_{\mathrm{S}}(r)}\right)^{2}$,

and by combining Eqs. (14) and (16), in virtue of the magnetic flux conservation, we obtain the final relation,

$\frac{\hat{B}_{\mathrm{S}}(r)}{\hat{B}_{\mathrm{N}}(r)} \approx\left(\frac{a_{\mathrm{N}}(r)}{a_{\mathrm{S}}(r)}\right)^{2}$,

which links the estimated deflections with the ratio of the magnetic field magnitudes. The squared values for the ratio $a_{\mathrm{N}} / a_{\mathrm{S}}$ at $r=2 R_{\odot}$, according our analysis of the jet deviations, are around 1.5-2.0 (see Table 1). These values are almost 25-65\% larger than the ratio of the magnetic field estimated by Erdos $\&$ Balogh (2010), in agreement with the larger estimate of the HCS offset in our analysis. The agreement between our results and those from Erdős \& Balogh (2010) are rather satisfactory. We would like to outline the comparison is made on the basis of different observables (jet deflection against interplanetary magnetic field measurements normalised to $1 \mathrm{AU}$ ) and refer in part to different periods in time. In addition, a list from WSO of the tilt of the HCS reports values greater than $10 \mathrm{deg}$ for the period of interest.

Therefore, we have an independent indication that the solar magnetic field can indeed exhibit a N-S asymmetry, a result that can have profound implications on the models of solar dynamo. The forthcoming missions Solar Probe Plus and Solar Orbiter will have a crucial role in assembling the extent of the N-S asymmetry more precisely, thanks to both in situ measurements of the nearly coronal magnetic field and remote observations. In particular, the Solar Orbiter UV instruments will allow us to more 
accurately check the size of the polar coronal holes and the deflection of polar jets, allowing us to better constrain the solar magnetic field.

Acknowledgements. We would like to thank the referee, Bernd Inhester, for his useful and fruitful comments. Data are courtesy of the STEREO/SECCHI team and the Wilcox Solar Observatory. G.N. and V.M.N. thank support from STFC consolidated grant ST/L000733/1. S.P. acknowledges support from the European Union (European Social Fund - ESF) and Greek national funds through the Operation Program "Education and Lifelong Learning" of the National Strategic Reference Framework (NSRF) - Research Funding Program: Thales. Investing in knowledge society through the European Social Fund, as well as from an FP7 Marie Curie Grant (FP7-PEOPLE-2010-RG/268288). V.B. acknowledges support from the German Space agency DLR through the project CGAUSS. CGAUSS (Coronagraphic German And US Solar Probe Plus Survey, Grant 50 OL1201) is the German contribution to the WISPR camera currently under development for the NASA SPP mission. V.M.N. work is further supported by the European Research Council under the SeismoSun Research Project No. 321141, and the BK21 plus program through the National Research Foundation funded by the Ministry of Education of Korea.

\section{References}

Altschuler, M. D., \& Newkirk, G. 1969, Sol. Phys., 9, 131

Aschwanden, M. J. 2005, Physics of the Solar Corona. An Introduction with Problems and Solutions, 2nd edn.

Bazilevskaya, G., Broomhall, A.-M., Elsworth, Y., \& Nakariakov, V. M. 2014, Space Sci. Rev., 186, 359

Bosman, E., Bothmer, V., Nisticò, G., et al. 2012, Sol. Phys., 281, 167

Bravo, S., \& González-Esparza, J. A. 2000, Geophys. Res. Lett., 27, 847
Bravo, S., \& González-Esparza, J. A. 2001, Geophys. Res. Lett., 28, 1667

Cremades, H., \& Bothmer, V. 2004, A\&A, 422, 307

de Patoul, J., Inhester, B., \& Cameron, R. 2013a, A\&A, 558, L4

de Patoul, J., Inhester, B., Feng, L., \& Wiegelmann, T. 2013b, Sol. Phys., 283, 207

Erdős, G., \& Balogh, A. 1998, Geophys. Res. Lett., 25, 245

Erdős, G., \& Balogh, A. 2010, J. Geophys. Res. (Space Phys.), 115, 1105

Fisk, L. A. 1996, J. Geophys. Res., 101, 15547

Heber, B., Droege, W., Ferrando, P., et al. 1996, A\&A, 316, 538

Hoeksema, J. T. 1995, Space Sci. Rev., 72, 137

Kivelson, M. G., \& Russell, C. T. 1995, Science, 269, 862

Markwardt, C. B. 2009, in Astronomical Data Analysis Software and Systems XVIII, eds. D. A. Bohlender, D. Durand, \& P. Dowler, ASP Conf. Ser., 411, 251

Mursula, K., \& Hiltula, T. 2004, Sol. Phys., 224, 133

Nakariakov, V. M., \& Ofman, L. 2001, A\&A, 372, L53

Nisticò, G., Bothmer, V., Patsourakos, S., \& Zimbardo, G. 2009, Sol. Phys., 259, 87

Nisticò, G., Verwichte, E., \& Nakariakov, V. 2013, Entropy, 15, 4520

Simpson, J. A., Zhang, M., \& Bame, S. 1996, ApJ, 465, L69

Tokumaru, M., Fujiki, K., \& Iju, T. 2015, J. Geophys. Res. (Space Phys.), 120, 3283

Vecchio, A., \& Carbone, V. 2008, ApJ, 683, 536

Vecchio, A., Laurenza, M., Meduri, D., Carbone, V., \& Storini, M. 2012, ApJ, 749,27

Virtanen, I. I., \& Mursula, K. 2010, J. Geophys. Res. (Space Phys.), 115, 9110

Wang, Y.-M. 1996, ApJ, 456, L119

Wang, Y.-M., \& Sheeley, Jr., N. R. 1992, ApJ, 392, 310

Wiegelmann, T., \& Sakurai, T. 2012, Liv. Rev. Sol. Phys., 9, 5

Yokoyama, T., \& Shibata, K. 1995, Nature, 375, 42 